TENDENCIAS

Revista de la Facultad de Ciencias

Económicas y Administrativas.

Universidad de Nariño

ISSN-E 2539-0554

Vol. XXI No. 2 - 2do Semestre 2020

Julio-Diciembre - Páginas 242-265

\title{
RELACIONAMIENTO DE MARCA: ANÁLISIS DE PERSPECTIVAS Y TRASCENDENCIA EN LA GESTIÓN
}

\section{BRAND RELATIONSHIP: PERSPECTIVES ANALYZES AND TRANSCENDING IN MANAGEMENT}

\section{RELACIONAMENTO DE MARCA: ANÁLISE DE PERSPECTIVAS E TRANSCENDÊNCIA EM A GESTÃO}

\author{
MEJÍA_Juan Camilo; ARCOS ROSAS_ Mario Fernando
}

Magíster en Mercadeo, Universidad de Nariño, Colombia. Estudiante de Doctorado en Marketing, Universidad de Valencia, España. E-mail: mejuanca@alumni.uv.es, España.

Magíster en Mercadeo, Universidad de Nariño, Colombia. Docente Departamento de Comercio Internacional, Universidad de Nariño. E-mail: mfarcosr@udenar.edu.co, Colombia.

Recibido: 29 de marzo de 2020

Aprobado: 4 de junio 2020

DOI: https://doi.org/10.22267/rtend.202102.149

\section{RESUMEN}

El presente artículo de revisión, describe y analiza el estado de la investigación concerniente al Relacionamiento de Marca (Brand Relationship) en el mundo, a partir de la exploración de artículos en inglés publicados en revistas que están catalogadas en ABI Inform (Proquest), Web of Science (WoS) y Scopus (Elsevier) en el periodo comprendido entre 2000 y 2020. Se construyó una base de datos de aproximadamente 143 artículos con el año en que fue publicado el documento, el autor, el nombre de la revista, el país y el método empleado en el estudio. Se puede observar luego de la revisión que en los últimos años ha tomado fuerza el tema y existe una proliferación de artículos relacionados directamente con el campo tratado o con sus dimensiones, sin embargo, empiezan a existir controversias sobre postulados previos de autores renombrados en el tema. De esta forma se discuten 
las diferentes dimensiones del tópico junto con su concepto principal, lo que se está investigando y el aporte que esto tiene para los gerentes de marca, concluyendo con algunas brechas de investigación futuras.

Palabras clave: relacionamiento de marca; personalidad de marca; gestión de marca; dimensiones de marca; identidad de marca; valor de marca.

JEL: M3; M30; M31; M32; M37; M39

\section{ABSTRACT}

This review describes and analyzes the state of research about Brand Relationship in the world, based on a review of articles in English published in journals that are listed in ABI Inform (Proquest), Web of Science (WoS) and Scopus (Elsevier) in the period from 2000 to 2020. A database of approximately 144 articles was built from the year the article, author, journal name, country, and method were published. employed in the study. It can be seen after the review that in recent years the topic has taken hold and there is a proliferation of articles related directly to the field treated or to its dimensions, however, there are directly controversies about previous postulates by renowned authors on the subject. The different dimensions of the topic are discussed together with its main concept, what is being investigated and the contribution this has for brand managers, concluding with some future research gaps.

Keywords: brand relationship; brand personality; brand management; brand dimensions; brand identity; brand equity.

JEL: M3; M30; M31; M32; M37; M39

\section{RESUMO}

Este artigo de revisão descreve e analisa o estado da pesquisa sobre Relacionamento da Marca, com base em uma revisão de artigos em inglês publicados em periódicos listados em ABI Inform (Proquest), Wos of Science (WoS) e Scopus (Elsevier) no período de 2000 a 2020. Foi construída uma base de dados com aproximadamente 144 artigos com ele ano em que foram publicados o documento, autor, nome da revista, país e método empregado no estudo. Observa-se, após a revisão, que nos últimos anos o tema se consolidou e há uma proliferação de artigos relacionados diretamente ao campo 
tratado ou a suas dimensões, no entanto, há controvérsias diretamente sobre postulados anteriores de autores renomados sobre o assunto. As diferentes dimensões do tópico são discutidas juntamente com seu conceito principal, o que está sendo investigado e a contribuição que isso tem para os gerentes de marca, concluindo com algumas lacunas de pesquisas futuras.

Palavras-chave: relacionamento da marca; personalidade da marca; gerenciamento de marca; dimensões da marca; identidade da marca; valor da marca.

JEL: M3; M30; M31; M32; M37; M39

\section{INTRODUCCIÓN}

El relacionamiento de marca es un tema que hoy en día atrae a muchos investigadores para intentar explicar algunas de las dimensiones que los consumidores y las empresas tienen en cuenta a la hora de relacionarse entre sí. Bajo la comprensión, que este proceso no es siempre satisfactorio se hace importante revisar algunas teorías, modelos y conceptos de las dimensiones que lo componen. Entre las que se abordan para este estudio resulta interesante el alcance que pueden tener para las organizaciones y los estrategas de marketing, las formas como pueden aprovecharlas en su quehacer diario, lo que ha motivado la atención creciente en la literatura académica (Reimann, Castaño, Zaichkowsky y Bechara, 2012; Papista y Dimitriadis, 2012; Park, Eisingerich y Park 2013; Kumar y Kaushik, 2020), debido principalmente a la coherencia entre la construcción de relaciones de marca y la necesidad de profundizar cada vez en su análisis.

Se asume que cualquier trabajo de revisión pretende recopilar la información más relevante sobre un tema específico (Knopf, 2006), este artículo describe los estudios que se han realizado sobre el Relacionamiento de Marca (año, revista científica, método y principales tópicos trabajados) con la intención de presentar una síntesis clara del estado del arte que pueda ser útil para gerentes de marca, docentes del área y estrategas de Marketing que estén interesados en ahondar más sobre la temática y que pueda ser un referente para futuras investigaciones sobre el Relacionamiento de Marca.

Para cumplir el objetivo mencionado se revisaron 143 artículos, los cuales se categorizaron en temáticas como Identidad de Marca, Imagen de Marca, Arquitectura de Marca, Gestión de Marca, Personalidad de Marca, Comunicaciones de la Marca y Efectos del Relacionamiento de Marca (Figura 
1) para ser comentados en el desarrollo de este documento. En primera instancia se plantea la metodología, seguida de la discusión sobre cada uno de los aspectos a tratar, los cuales se dividen en una parte conceptual, para posteriormente incluir algunos comentarios sobre el tema, junto con un pequeño aporte o idea para los gerentes o encargados de marca y se cierra el documento planteando futuras líneas de investigación y las respectivas referencias que se tuvieron en cuenta para este documento.

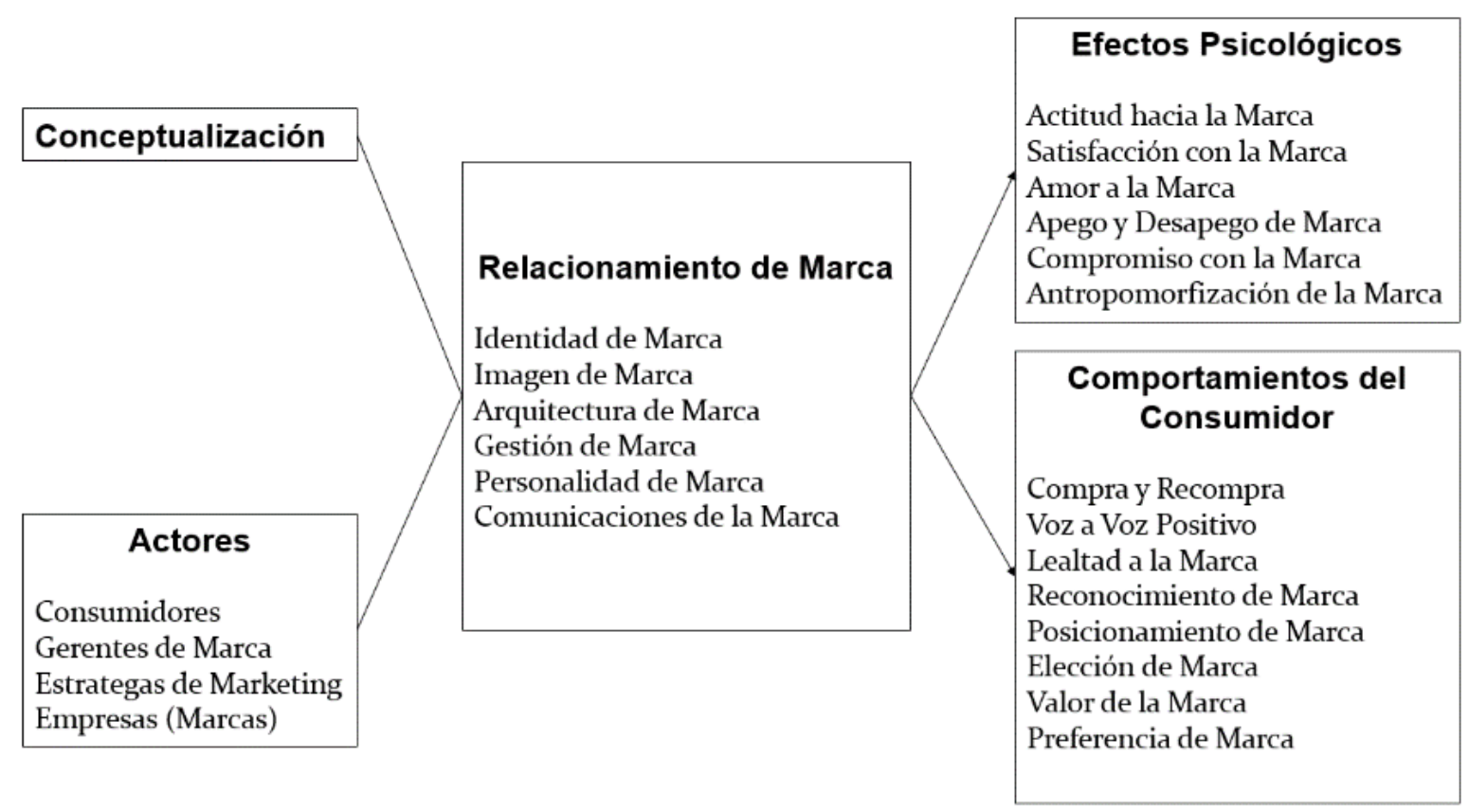

Figura 1. Temas tratados sobre Relacionamiento de Marca en el artículo.

Fuente: elaboración propia, 2020.

\section{REFERENTES TEÓRICOS}

Para la construcción de este breve marco teórico se conceptualiza únicamente acerca de Relacionamiento de Marca debido a que en el apartado donde se presenta la discusión y los resultados se introducen otros conceptos sobre el tema. En este sentido, se presentan los principales exponentes de la temática.

El Relacionamiento de Marca se define como la relación consumidor-marca que vincula una persona con una marca, aplicado de manera interdependiente y voluntaria entre las partes. Este concepto no es novedoso y es fácilmente entendible a través de una analogía con la relación compleja de 
conocimientos, afectos y comportamientos entre dos personas que en este caso serían la marca y el consumidor. Una relación entre estos resulta de la acumulación de experiencias de consumo y para que está exista evidentemente debe haber una interdependencia entre las partes, es decir, los dos deben afectar, definir y redefinir colectivamente dicha relación (Blackston, 1992, 1993; Fournier, 1998).

Antes de iniciar el siglo XXI, Fournier (1998) sugirió que los consumidores percibían una marca como una entidad que generaba algún tipo de conducta. La propuesta básica era construir relaciones entre el consumidor y la marca, lo que suponía que los consumidores tradujeran el comportamiento de una marca al lenguaje comprensible para ellos, en cierta medida las marcas se comunican con sus consumidores y generan relaciones entre las partes (Bengtsson, 2003). El Relacionamiento de Marca ha llegado a una nueva etapa para ser uno de los principales objetivos de investigación sobre consumidores y marcas (Aaker, Fournier y Brasel, 2004; Chang y Chieng, 2006; Breivik y Thorbjørnsen, 2008).

\section{METODOLOGÍA}

El método de investigación empleado fue la revisión de literatura. De acuerdo con Knopf (2006) es un método sistemático que tiene por objetivo identificar, evaluar y sintetizar la existencia de trabajos relacionados con el tópico en cuestión y que ha sido producido por expertos, investigadores y/o académicos. Lo primero que presenta es un resumen descriptivo de artículos que tratan sobre el relacionamiento de marca para luego analizar bajo unas categorías los avances que se han realizado y hacia donde pretende evolucionar esta temática. Para esta revisión se adopta el modelo propuesto por Mayring (2003) en cuatro pasos: 1) Recolección de material: Este es definido y delimitado para artículos de investigación en inglés. 2) Análisis descriptivo: Se describen los aspectos formales de las publicaciones. 3) Selección de categorías: Se seleccionan las dimensiones estructurales y las categorías de análisis que se aplicaron en la recolección de material. 4) Evaluación del material: Se analiza el material de acuerdo a las dimensiones con lo que se podría identificar e interpretar los resultados relevantes. 


\section{DISCUSIÓN}

\section{Recolección de material}

Para este ejercicio se utilizaron los catálogos digitales Web of Science (WoS), Scopus (Elsevier), ABI Inform (ProQuest), en los cuales se buscaron artículos científicos de los últimos 20 años hasta lo trabajado en el 2020, bajo la palabra clave en el título de la publicación "Brand Relationship" y que hagan parte de revistas científicas cuyo campo principal sea el Marketing. En la Figura 2 se relacionan los hallazgos.

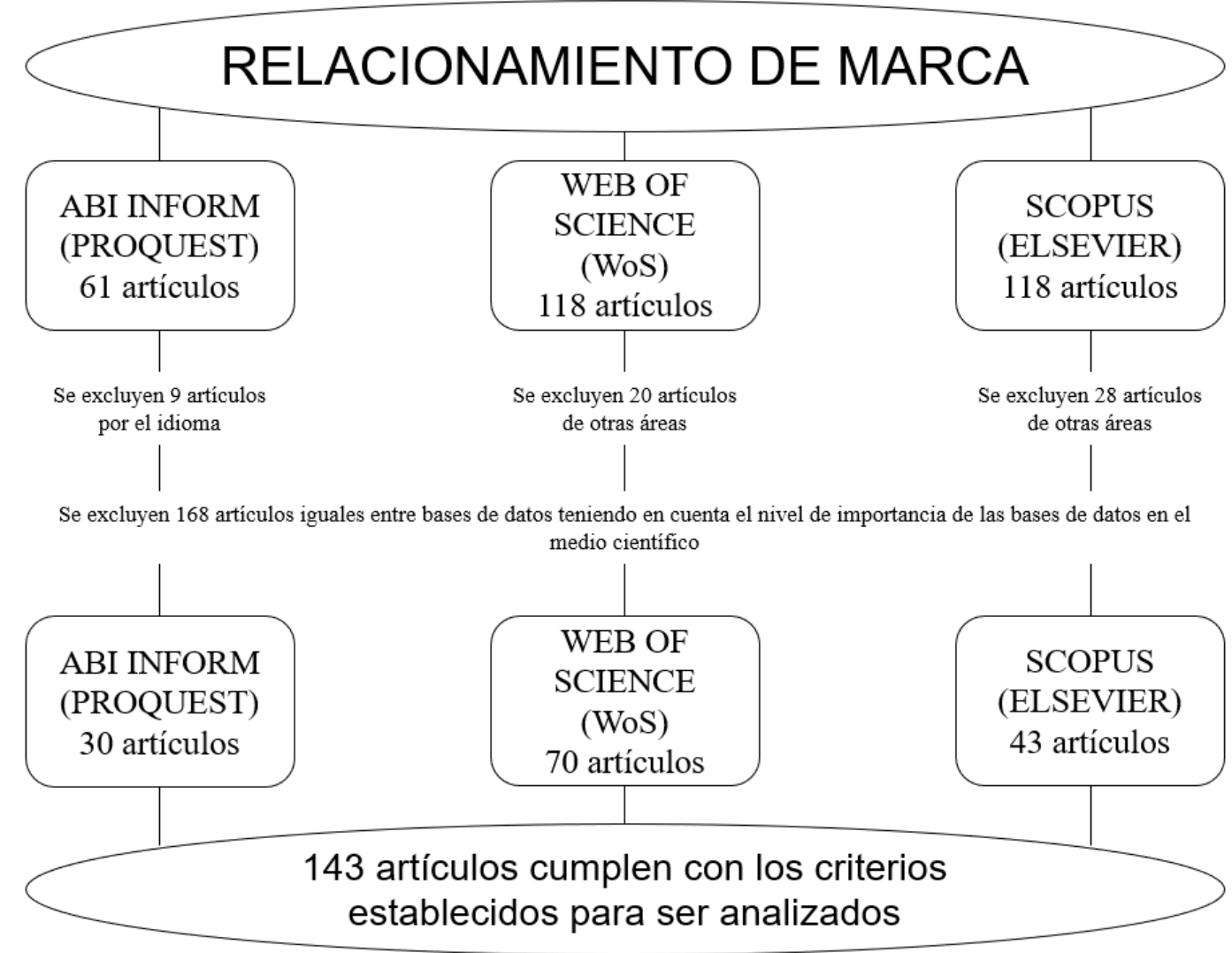

Figura 2. Material recolectado.

Fuente: elaboración propia, 2020.

En la búsqueda se encontraron un total de 368 artículos en los tres catálogos, para evitar repetición se descartaron 168 artículos teniendo como único criterio la relevancia de la base de datos en el medio científico y académico, además, 9 que no cumplían el requisito del idioma inglés en ABI Inform, 20 
que eran de otras áreas de la Web of Science y 28 de Scopus por la misma razón, dejando un total de 143 artículos que se organizaron en un archivo, con los cuales se desarrolló la siguiente fase descriptiva que se presenta en las tablas y figuras consecuentes.

\section{Análisis descriptivo}

Los artículos objeto de análisis (143), se clasificaron con base en el año de publicación, autores y la revista científica en el cual está publicado (Tabla 1), además se presenta una figura con los años de clasificación, el número de artículos y de autores (Figura 3), en la que se puede observar un aumento significativo entre los años 2012 a 2016, siendo el más productivo el 2014 en ese lapso de tiempo, luego se presenta una baja en el 2017 cuya razón no hace parte de esta revisión y, para el 2018 y el 2019 se presenta el máximo número de investigaciones pudiéndose notar en la figura claramente. Por último, con respecto al año 2020, existe una incertidumbre en si continuará una tendencia alcista o el número de publicaciones se verá reducido por lo que actualmente sucede, sin embargo, queda claro el interés de los autores por este tema. 


\section{Tabla 1}

Clasificación de artículos por año, autores y journal

\begin{tabular}{|c|c|c|c|c|}
\hline Año & $\begin{array}{l}\text { Número de } \\
\text { Artículos }\end{array}$ & $\begin{array}{l}\text { Numero } \\
\text { de autores }\end{array}$ & Autores & Revistas Científicas \\
\hline 2000 & 3 & 4 & Aaker y Joachimsthaler (2000); Blackston (2000); Kates (2000) & $\begin{array}{l}\text { California Management Review (1), Journal of Advertising Research (1), } \\
\text { Psychology and Marketing (1) }\end{array}$ \\
\hline 2002 & 1 & 4 & Thorbjørnsen, Supphellen, Nysveen y Pedersen (2002) & Journal of Interactive Marketing (1) \\
\hline 2003 & 2 & 2 & Wyner (2003); Kates (2003) & Marketing Management (1), Advances in Consumer Research (1) \\
\hline 2004 & 1 & 1 & Aggarwal (2004) & Journal of Consumer Research (1) \\
\hline 2005 & 3 & 9 & $\begin{array}{l}\text { Jevons, Gabbott y de Chernatony (2005); Nysveen, Pedersen, Thorbjørnsen y } \\
\text { Berthon (2005); Hess y Story (2005) }\end{array}$ & $\begin{array}{l}\text { Journal of Product and Brand Management (1), Journal of Service Research (1), } \\
\text { Journal of Consumer Marketing (1) }\end{array}$ \\
\hline 2006 & 4 & 13 & $\begin{array}{l}\text { Pao-Long,y Ming-Hua (2006); Heath, Brandt y Nairn (2006); Beverland, Lim, } \\
\text { Morrison y Terziovski (2006); Hayes, Alford, Silver y York (2006) }\end{array}$ & $\begin{array}{l}\text { Psychology y Marketing (1), Journal of Advertising Research (1), Journal of } \\
\text { Business Research (1), Journal of Product and Brand Management (1) }\end{array}$ \\
\hline 2007 & 4 & 7 & Swaminathan, Page y Gurhan-Canli (2007); Zhou (2007); Kang, Kim y Shin (2007) & $\begin{array}{l}\text { Journal of Consumer Research (1), Frontiers of Business Research in China (1), } \\
\text { Journal of Global Scholars of Marketing Science (1) }\end{array}$ \\
\hline 2008 & 3 & 8 & $\begin{array}{l}\text { Busacca, Pelloni y Bertoli (2008), Breivik y Thorbjørnsen (2008); Punjaisri, Wilson } \\
\text { y Evanschitzky (2008) }\end{array}$ & $\begin{array}{l}\text { Esic Market (1), Journal of the Academy of Marketing Science (1), Journal of } \\
\text { Relationship Marketing (1) }\end{array}$ \\
\hline 2009 & 5 & 6 & $\begin{array}{l}\text { Sung y Campbell (2009); Veloutsou y Moutinho (2009); Bowden (2009); Commuri } \\
\qquad(2009)\end{array}$ & $\begin{array}{c}\text { Journal of Public Policy and Marketing (1), Journal of Brand Management (1), } \\
\text { Journal of Business Research (1), Journal of Hospitality and Leisure Marketing (1), } \\
\text { Journal of Marketing }\end{array}$ \\
\hline 2010 & 5 & 13 & $\begin{array}{c}\text { Huang, Wang y Zhou (2010); Sahay y Sharma (2010); Odekerken-Schröder, } \\
\text { Hennig-Thurau y Knaevelsrud (2010); Jillapalli y Wilcox (2010); Ellonen, } \\
\text { Tarkiainen y Kuivalainen (2010) }\end{array}$ & $\begin{array}{l}\text { Psychology y Marketing (1),Journal of Business Research (1), Journal of Retailing } \\
\text { (1), Journal of Marketing Education (1), International Journal of Internet Marketing } \\
\text { and Advertising (1) }\end{array}$ \\
\hline 2011 & 5 & 9 & $\begin{array}{l}\text { Nguyen (2011); Sarkar (2011); Zayer y Neier (2011); Nyadzayo, Matanda y Ewing } \\
\text { (2011); Belaid y Behi (2011) }\end{array}$ & $\begin{array}{c}\text { Journal of Relationship Marketing (1), Management y Marketing (1), Qualitative } \\
\text { Market Research (1), Industrial Marketing Management (1), Journal of Product and } \\
\text { Brand Management (1) }\end{array}$ \\
\hline 2012 & 11 & 26 & $\begin{array}{l}\text { Avis, Aitken y Ferguson (2012); Papista y Dimitriadis (2012); Aurier y Gilles } \\
\text { (2012); Sahay, Sharma y Mehta (2012), Cheng, White y Chaplin (2012); Zhou, } \\
\text { Zhang, Su y Zhou (2012); Reimann, Castaño, Zaichkowsky y Bechara (2012); } \\
\text { Magnoni (2012); Huang (2012); Hwang y Kandampully (2012); Keller (2012) }\end{array}$ & $\begin{array}{l}\text { Marketing Theory (1), Qualitative Market Research (1), European Journal of } \\
\text { Marketing (1), Journal of Brand Management (2), Journal Of Consumer } \\
\text { Psychology (3), Journal of Business Research (1), Advances in Consumer Research } \\
\text { (1), Journal of Product and Brand Management (1) }\end{array}$ \\
\hline
\end{tabular}




\section{Pentina, Gammoh, Zhang y Mallin (2013); Park, Eisingerich y Park (2013);}

Morgan-Thomas y Veloutsou (2013); Alba y Lutz (2013); Tasiyana y Rowley (2013); Lambert y Desmond (2013); Schmitt (2013); Lee y Kang (2013); Bennett, Hill y Oleksiuk (2013); Albert y Merunka (2013); Tuškej, Golob y Podnar (2013);

Sreejesh (2014), Zainol, Yasin, Omar y Hashim (2014), Lo y Im (2014), Kim y Cho (2014), Yang y Tang (2014), Loureiro (2014), Manarioti y Kaufmann (2014), Fritz, Lorenz y Kempe (2014), Ramaseshan y Stein (2014), Hanslin y Rindell (2014), Ahmad y Thyagaraj (2014), Cayolla y Loureiro (2014), Labrecque (2014), Kemp, Jillapalli y Becerra (2014), Carnevale, Yucel-Aybat y Block (2014), Fetscherin, Boulanger, Filho y Souki (2014), Leung, Bougoure y Miller (2014), Huang y Mitchell (2014),

Michel, Merk y Eroglu (2015), Mogos y Pauwels-Delassus (2015),Todd (2015), Guo y Zhang (2015), Avis y Aitken (2015), Fetscherin y Heinrich (2015), Hodge, Romo, Medina y Fionda-Douglas (2015), Kessous, Roux y Chandon (2015), Sajtos, Kreis y Brodie (2015), Kamath y Aggarwal (2015), Hongcharu y Eiamkanchanala (2015)

Mishra (2016), Tho, Trang y Olsen (2016), Nyadzayo, Matanda y Ewing (2016), Rodhain y Aurier (2016), Zainol, Omar, Osman y Habidin (2016), Ghantous (2016), Trudeau y Shobeiri (2016), Confos y Davis (2016), Arikan, Yilmaz y Bodur (2016), Ramaswami, Raju y Page (2016), Chiu y Won (2016), KarpińskaKrakowiak (2016)

Ramadan (2017), Hsu y Liou (2017), Hawkins (2017), Ferguson, Brown y Johnston (2017), Muroyiwa, Abratt y Mingione (2017)

Ahn y Back (2018); Seric y Pranicevic (2018); Casidy, Wymer y O'Cass (2018); Fazal-e-Hasana, Ahmadi, Mortimer, Grimmer y Kelly (2018); Charton-Vachet y Lombart (2018); Martin (2018); Consiglio, Kupor, Gino y Norton (2018); Kumar y Nayak (2018); Ghani y Tuhin (2018); Giovanis y Athanasopoulou (2018); Jain, Kamboj, Kumar y Rahman (2018); Voorn, van der Veen, van Rompay y Pruyn

\section{Academy of Marketing Studies Journal (1), Journal of Consumer Psychology (3),}

Journal of Business Research (3), Psychology and Marketing (1), Academy of Marketing Studies Journal (1), Journal of Public Policy and Marketing (1), Journal of Consumer Marketing (1)

Journal of Relationship Marketing (1), Journal of Travel y Tourism Marketing (1), Asia Pacific Journal of Tourism Research (1), International Conference On Service Systems And Service Management (1), Future Of Entrepreneurship (1), Journal Of Retailing And Consumer Services (1), Psychology and Marketing (2), Journal of Brand Management (3), Journal of Fashion Marketing and Management (1), International Journal of Hospitality Management (1), International Journal of Business and Globalisation (1), Journal of Interactive Marketing (1), Journal of

Services Marketing (1), Journal of Product and Brand Management (1)

The Journal of Personal Selling y Sales Management (1), The Journal of Consumer Marketing (2), Academy Of Marketing Science Annual Conference (1), Journal of Historical Research in Marketing (1), Journal of Business Research (1), Journal of Brand Management (1), Psychology and Marketing (1), Journal of Service Theory and Practice (1), Emerald Emerging Markets Case Studies (1), International Journal of Economic Research (1)

Journal of Business Research (1), Asia Pacific Business Review (1), Industrial Marketing Management (1), The Journal of Product and Brand Management (1), Journal of Relationship Marketing (1), Services Marketing Quarterly (1), The Journal of Product and Brand Management (1), European Journal of Marketing (1), Journal Of Business Economics And Management (1), Journal of Indian Business Research (1), Journal of Product and Brand Management (1), International Journal of Research in Marketing (1)

Qualitative Market Research (1), International journal of mobile communications (1), Journal of Brand Management (1), Journal of Business Research (1), South African Journal of Business Management (1)

Journal of Travel y Tourism Marketing (1), Journal Of Hospitality Marketing y Management (1), Tourism Management (1), Journal Of Retailing And Consumer Services (2), Journal Of Strategic Marketing (1), Journal of Consumer Psychology (2), Journal of Retailing and Consumer Services (1), International Journal of Supply 
Relacionamiento de marca: análisis de perspectivas y trascendencia en la gestión Mejía_Juan Camilo; Arcos Rosas_ Mario Fernando

(2018); Frizzo, Korelo y Prado (2018); Papista, Chrysochou, Krystallis y Dimitriadis (2018); Scholz y Duffy (2018)

Erkmen y Hancer (2019); Pulh, Mencarelli y Chaney (2019); Pourazad, Stocchi y Pare (2019); Hashim, Yasin (2019); Hashim y Yasin (2019); Carlson, Wyllie, Rahman y Voola (2019); Khamitov, Wang y Thomson (2019); Nobre y Simoes (2019); Wang y Lang (2019); Hashim y Yasin (2019); Chankoson y Thabhiranrak

(2019); Lee y Jin, (2019); Langaro, Salgueiro, Rita y Del Chiappa (2019); Pawar y Raut (2019); Reckmann y Teichert (2019); Mena, Hult, Ferrell y Zhang (2019);

Fernandes y Moreira (2019); Eggert, Steinhoff y Witte (2019); Fetscherin, Guzman, Veloutsou y Cayolla (2019); Lopez, Llonch y Rialp (2019); Jain y Sharma (2019); Oh, Prado, Korelo y Frizzo (2019)

2020 4 11

Ebrahimi, Sadeghvaziri y Abyaneh (2020); Hashim, Yasin y Ya'kob (2020); Kumar y Kaushik (2020); Liu, Yang y Chen (2020)
Chain Management (1), Journal of Retailing and Consumer Services (2), Journal of Consumer Behaviour (2), Journal of Service Theory and Practice (1)

International Journal of Contemporary Hospitality Management (1), European Journal of Marketing (1), Australasian Marketing Journal (1), Journal Of Retailing And Consumer Services (1), Journal Of Consumer Research (1), Journal Of Retailing And Consumer Services (1), Polish Journal Of Management Studies (1), Sustainability (1), Journal Of Creative Communications (1), Indian Journal of Marketing (1), Marketing, Zeitschrift fur Forschung und Praxis (1), Journal of Business Research (3) Journal of Product and Brand Management (3), Journal of Marketing (1), Spanish Journal of Marketing - ESIC (1), Journal of Consumer Marketing (1)

Iranian Journal Of Management Studies (1), Journal Of Marketing For Higher Education (1), Journal of Strategic Marketing (1), Sustainability (1)

Fuente: elaboración propia, 2020. 


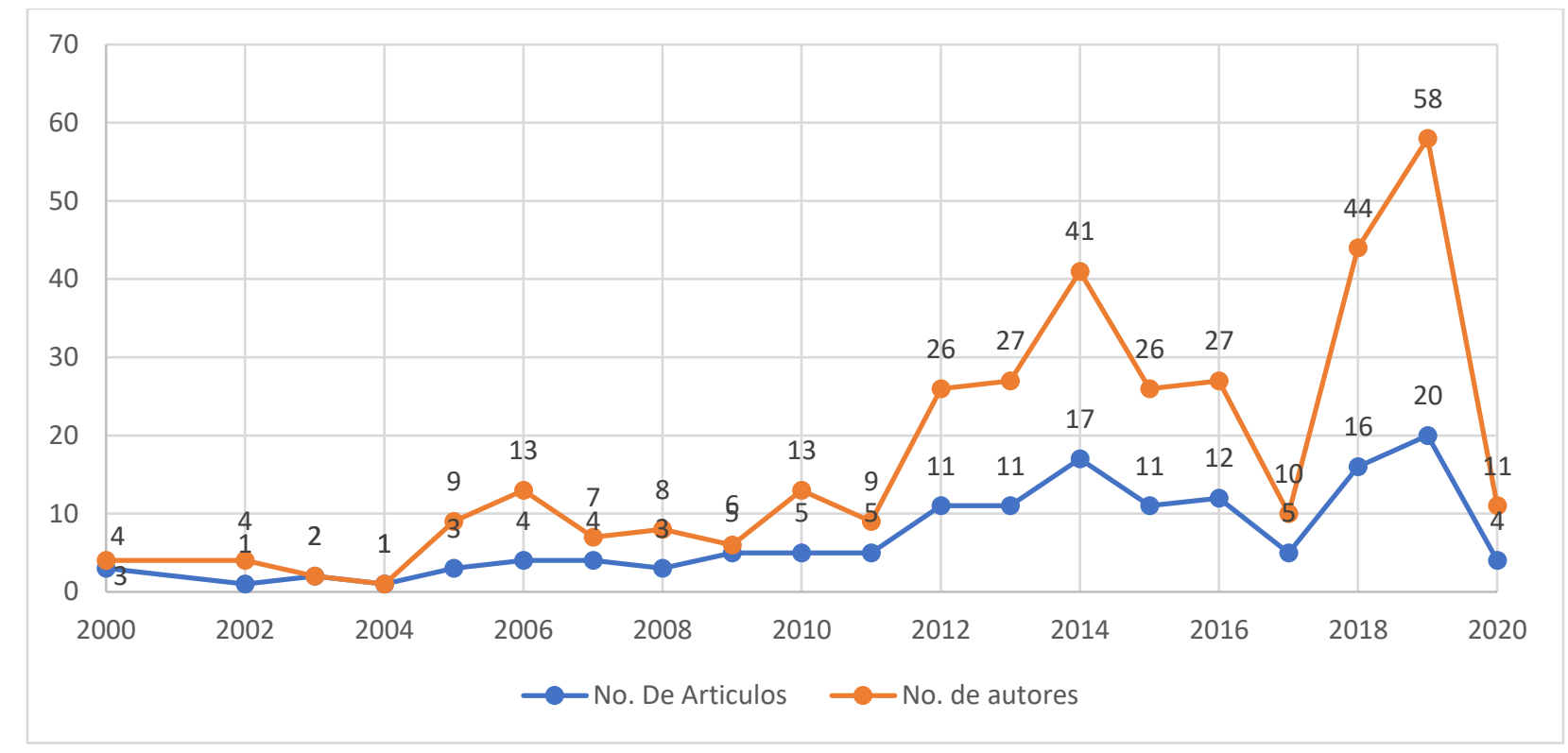

Figura 3. No. de Artículos y Autores 2000 - 2020.

Fuente: elaboración propia, 2020.

De igual manera, se puede observar (Tabla 1) que los estudios se extendieron en 44 revistas internacionales diferentes. La mayoría de estos se publicaron en las siguientes revistas: Journal of Brand Management, Journal of Business Research, Journal of Consumer Psychology y Journal of Relationship Management. Si bien una gran cantidad se editan en Reino Unido (UK) 47\% y Estados Unidos (USA) 38\%, resulta interesante la aparición de China con un 9\% del total de publicaciones y otros países como Chile, Sudáfrica, India, Malasia y Taiwán con un $1 \%$ cada uno. En cuanto al método utilizado para la investigación el $73 \%$ lo hizo de forma cuantitativa, el 13\% cualitativa, el $8 \%$ experimental, el 5\% revisión de literatura y el 1\% mixto, esto tiene una clara explicación al remitirse a Bartels (1951) sobre la necesidad de convertir el marketing en una ciencia, este autor afirma que para lograrlo, es importante establecer leyes generales verificables, principalmente a través de un proceso hipotético, que permita sistematizar y formular verdades generalizables basadas en percepciones, las cuales respondan a la búsqueda, entendimiento y comprensión de las realidades de los diferentes entornos. 


\section{Selección de categorías y evaluación del material}

Como se mencionó previamente en la Figura 1, se seleccionaron diversos temas partiendo de las investigaciones que se han publicado a lo largo de los 20 años evaluados, si bien la marca es un concepto que se fortalece con la llegada del consumo de masas, cuando éstas buscaban diferenciar a las empresas de la competencia, esta depende radicalmente del contexto desde donde se analice. Es importante aclarar que los artículos revisados sobre relacionamiento de marca convergen en opiniones más o menos similares, por ello resulta interesante hablar de algunos a manera de comentario según los diferentes artículos descritos.

Identidad de marca: La identidad de marca es un conjunto único de asociaciones con la marca que implica una promesa a los clientes e incluye una identidad central y extendida. La identidad central es la esencia principal e intemporal de la marca que permanece constante a medida que la marca se traslada a nuevos mercados y nuevos productos y, hace relación a los atributos del producto, el servicio, el perfil del usuario, el ambiente de la tienda, entre otros. La identidad extendida se presenta en torno a elementos de identidad de marca organizados en grupos coherentes y significativos que proporcionan integridad a la marca, y se enfoca en la personalidad, la relación y la fuerte asociación con los símbolos de la marca. Dichos elementos son la presentación, posicionamiento, personalidad, visión de la marca, cultura, valores, modelos mentales y relacionamiento con cada uno de los stakeholders (Aaker y Joachimsthaler, 2000).

Cuando se usa la misma marca en productos, segmentos y países, generalmente se hacen dos suposiciones implícitas, las cuales son totalmente contrarias a la idea de identidad. La primera, es que puede haber diferentes identidades de marca y posiciones en cada contexto a pesar de la marca común, sin embargo, el uso de docenas de identidades de marca crea anarquía de marca y es una receta para la construcción de marcas ineficientes e ineficaces. La segunda suposición, es que existe una identidad y una posición única en todas partes, incluso, si la imposición de una identidad de una única marca conlleva un compromiso mediocre que es ineficaz en muchos de sus contextos. De hecho, generalmente debe haber un número limitado de identidades que comparten elementos comunes pero que tienen distinciones (Kumar y Kaushik, 2020; Aaker y Joachimsthaler, 2000). 
En resumen, las marcas ayudan a establecer y reforzar identidades deseadas, tanto para sus propios consumidores como para los demás a su alrededor (Zayer y Neier, 2011). El mercado en ese punto sirve como un lugar privilegiado en el que se buscan marcas para expresar significados culturales sobre algo que permita diferenciar una cultura de otra, esta información para Zhou (2007) debe ser útil para que el gerente de marca la considere como un símbolo con el cual se pudiera construir el marco de identificación entre la marca y el consumidor siendo consciente de la variedad de relaciones que la marca provee al usuario, donde resulta importante tener en cuenta el contexto en el que se circunscriben dichas relaciones.

Imagen de marca: Se define como un fenómeno subjetivo y perceptivo de marca que se refleja en una red de asociaciones en la memoria de los consumidores (Keller citado por Chang y Chieng, 2006), generalmente considerada como la suma de las asociaciones tangibles e intangibles de marca y las percepciones que los consumidores tengan de ella. La imagen de marca se basa no solo en la actitud de la misma sino también en su asociación, estas asociaciones conducen a la formulación de una imagen distinta en la mente de los consumidores.

En 2019, Erkmen y Hancer en una investigación para restaurantes turcos de lujo obtuvieron información que les permitió afirmar que crear un conjunto de asociaciones de marca es la esencia de crear una imagen de marca positiva, además de confirmar teorías anteriores donde la imagen de marca se componía específicamente de dos elementos, el primero, las asociaciones que los consumidores atribuyen a la marca y el segundo, la personalidad de ésta, por lo tanto, las mejoras en la fuerza, la favorabilidad y la unicidad de las asociaciones de marca mejorarán la imagen de la marca, mientras que su personalidad es un constructo que por muchos años ha inquietado a expertos de marketing y estudiosos del comportamiento, ya que se valida la 'antropomorfización' de la marca, jugando un papel clave en la creación de partidarios a humanizar las marcas convirtiendo la relación con ellas en una relación similar a la de amigos, familia o pareja.

Los autores mencionados concluyen su investigación generando propuestas para los encargados de la imagen de marca, entre ellas cabe resaltar la atención a los elementos físicos en las instalaciones, el diseño de los espacios, la debida segmentación del público atendido, la calidad en los productos, el servicio intachable y por último, la innovación que puede brindar la oportunidad de crear una imagen 
de marca única al ir más allá de la satisfacción, logrando un relacionamiento perfecto con la marca (Erkmen y Hancer, 2019; Kaufmann, Filho, Silva y Monteiro, 2019).

Arquitectura de marca: Aaker y Joachimsthaler (2000) crean el concepto argumentando que los que trabajan con las marcas enfrentan lo mismo que un arquitecto cuando debe planear la estructura y el diseño de habitaciones, edificios y ciudades, por lo cual, una arquitectura de marca coherente puede generar impacto, claridad, sinergia y apalancamiento en lugar de debilidad, confusión, desperdicio y pérdidas en el mercado. Además, cuando un producto se relaciona con otros de niveles superiores o inferiores y con otro tipo de marcas, crea su universo de relaciones que lo dota de significados profundos y condiciona las relaciones del producto con otras marcas externas e internas al contexto organizacional.

La coherencia, respaldo, conexión, vinculación, identidad y efectividad de la marca, van de la mano en la elección de una u otra arquitectura, entendida ésta como la cara externa de la estrategia del negocio, que debe alinearse perfectamente con los objetivos organizacionales, puesto que condiciona la creación de unidades estratégicas y por ende, la sustentabilidad de la compañía que de ella dependen, claro está, que la elección siempre estará supeditada a las percepciones que tienen los clientes, de allí la importancia de estructurar un diseño que unifique la estrategia de la compañía, sea ésta grande o pequeña, con las necesidades propias del mercado que atiende, afianzando e incrementando de esta manera su valor en el tiempo y gestionando sin descuido la relación que se logra con el consumidor (Papista y Dimitriadis, 2012; Aaker y Joachimsthaler, 2000).

Este último puede elegir una marca tanto por las experiencias internas como externas, aunque a menudo sin un contexto de exposición relacionado (Petromilli, Morrison y Million, 2002), por ello, los encargados de marca deben estar atentos según los autores, a esperar la respuesta perceptiva (¿Qué percibe el cliente?), de relacionamiento (¿Cómo se relaciona el cliente con la marca?) y de creación de valor (¿Cuál es el valor que la marca aporta a sus clientes?) y al obtener dichas contestaciones actuar ávidamente en relación a la competencia para realizar los ajustes a la estrategia inicial en busca de mantener y fortalecer su relación con el consumidor. 
Gestión de la marca: Es un campo de estudio relativamente joven y uno, en el que el interés real se mostró solo durante la última década del siglo anterior, momento en el que se formularon las teorías más importantes y se produjo la literatura más valiosa (Krake, 2005). Hay muchas definiciones, una de ellas sería el reconocimiento de una organización por ejecutar acciones estratégicas sobre la marca y desarrollar una serie de actividades para fortalecerla en el mercado sobreentendiendo que estos no son ejercicios únicos, sino, un aspecto recurrente de la política de marketing que se presenta bajo dos pilares, la diferenciación (carácter distintivo de un producto) y el valor agregado (cuando un producto tiene más valor para el cliente) con lo que en definitiva se llega a crear un relacionamiento con el consumidor (Keller citado por Krake, 2005).

La gestión de marca empieza desde la ideación del producto y la puesta en el mercado para el reconocimiento del público hacia el cual se diseñó, continua con el registro, donde legalmente obtiene un nombre, una identidad, una personalización, un ser y un quehacer, para seguir con los programas de marketing que buscan la asociación con la marca, aprovechando todos los elementos del marketing mix que deben estar claros y dirigidos por la estrategia de la organización que busca atraer el mayor número de consumidores en el mercado a partir de una oferta de valor diferenciada de la competencia (Nyadzayo et al., 2016).

Según los mismos autores, la gerencia de marca debe estudiar y conocer las necesidades del público objetivo, para ello sugieren una óptima recolección de información y un buen manejo de ésta, ya que gracias al conocimiento del público se divulga información pertinente bajo el contexto determinado; interactuando e interviniendo en la relación entre el consumidor y su marca y, respondiendo efectivamente a la inversión que puede mejorar el desempeño en cada área en relación con los costos y otras formas alternativas en que podrían gastar el dinero. Para terminar, es importante conectarse con el consumidor a partir de procesos vanguardistas que identifiquen a la marca con el usuario y fortalezcan día a día la relación que con éste tiene.

Personalidad de la marca: Desde 1955 Gardner y Levi afirmaban que las marcas tienen personalidades, como una metáfora de los atributos de marca intangibles. Ésta se puede definir como las características humanas asociadas con una marca (Aaker y Joachimsthaler, 2000), teóricamente la marca humanizada aparece por primera vez a principios de los 90`s pero fueron Aaker et al. (2004) 
quienes introdujeron el concepto de antropomorfismo al momento de atribuir características humanas a los no humanos, como una explicación provista desde la psicología para explicar este fenómeno. Sin embargo, según la revisión de literatura se encontró que, a pesar de dicha asunción, existen contradicciones para ello, puesto que son utilizadas por los investigadores como metáforas para explicar las percepciones de marca, ayudando con este lenguaje figurativo a la comprensión del comportamiento y la formación de relaciones que se presentan entre marcas y consumidores.

Varios autores en la literatura trabajan el tema de Personalidad de Marca, ya que fortalece la idea de que los consumidores tengan relaciones reales con las marcas (Avis y Aitken, 2015), entre esta mirada humana se destacan algunas figuraciones que la marca debe presentar para lograr esa relación entre las que sobresalen la sinceridad (honesta, coherente, atrevida), emoción (atrevida, enérgica, imaginativa, actualizada), competitividad (confiable, inteligente, exitosa) sofisticada (clase alta, encantadora), robusta (libre, dura). Todo este proceso de pretender que la marca tenga personalidad cambia los objetivos de la misma, de ser un simple nombre o logo a tener vida propia y de esta manera fortalecer o distorsionar las relaciones que continuamente se presentan.

Para los encargados de marca en las organizaciones es importante gestionar la Personalidad de la misma, intentando de manera clara que ésta genere una actitud y una relación positiva sobre el consumidor. Por su parte, Lee y Kang (2013) afirman que personalidades sinceras y bonitas actúan positivamente sobre la actitud de los consumidores con base en la confianza y el compromiso de la marca hacia ellos y que una personalidad fuerte y emocionante intenta atraer a aquellos que se visualizan de tal manera. Sin duda, las opiniones y percepciones de los consumidores sobre determinada marca le imprimen unos trazos a la relación que se pueden presentar entre las partes, por esta razón se debe manejar un propósito en la empresa de forma selectiva hacia determinado segmento.

Comunicaciones de la marca: Hace referencia a los medios conocidos en el mundo del Marketing como promoción de marca, entre estos se incluyen las ventas personales, publicidad, promoción de ventas y relaciones públicas, utilizados para comunicar información de la marca. La mayoría de las empresas utilizan una combinación de estos medios, sin embargo y de manera definitiva las características de los mercados son las que determinan cuáles son aquellos que resultan más ventajosos para lograr el objetivo de comunicación con el cliente, aparte de esto, la estrategia de Marketing 
necesita, para ser exitosa, construir y fortalecer las relaciones que son las que brindan una gran cantidad de contenido y permiten la masificación o viralización de los mensajes, logrando que la inversión en cualquier medio pueda crear la relación con el consumidor, aumentando la credibilidad, la influencia y la acción. (Karpińska, 2016).

Como se reconoce ampliamente, los diferentes mensajes comunicacionales tienen poderes persuasivos, por un lado, están los mensajes utilitarios que tienen un mayor impacto en el comportamiento de compra de los consumidores, mientras que los mensajes emocionales mejoran la formación de actitudes positivas hacia la marca (Heath et al., 2006; Karpińska, 2016). Según la revisión en este aspecto, el contenido del mensaje se aborda desde dos perspectivas: la emocional y la racional, la primera, intenta conectar con los sentimientos de los consumidores lo que implica enviar señales no necesariamente relacionadas con el producto, ya que lo que se pretende es construir cierta atmósfera alrededor de la marca para impulsar sentimientos hacia ella y la segunda, que busca accionar el comportamiento de ellos, para lo cual se describen principalmente los beneficios funcionales, aparte de factores como la calidad, fiabilidad, accesibilidad, utilidad y conveniencia. Es importante tener en cuenta que para los consumidores están presentes ideas relacionadas con el posicionamiento, reputación, valor y la representación que en ellos generan las interacciones que la marca tiene en distintos momentos (Heath et al., 2016; Hudson, Huang, Roth, y Madden, 2016).

Para vincular y lograr una comunicación de la marca con la sociedad, los gerentes de marketing deben considerar factores como:

- La participación directa donde la marca se involucra en actividades sociales.

- La participación indirecta donde se evita al máximo forzar las relaciones para que estas se presenten.

- La expresión de sentimientos y experiencias, que generan rasgos de simpatía por la sociedad.

- La consistencia que tiene como fin crear relaciones a través de una participación constante en diferentes momentos.

- La continuidad que permita a la sociedad entender el apoyo de la marca hacia ella. Y,

- La extensión de la comunicación por parte de los mismos consumidores en su contexto. 
Cuando los encargados de la marca la presentan en sociedad, deben afinar de manera clara y coherente los mensajes apropiados, definiendo los medios adecuados, mejorando así el compromiso de la marca con la sociedad y ayudando a crear relaciones con los miembros de la misma (Hongcharu y Eiamkanchanalai, 2015; Karpińska, 2016).

Efectos del relacionamiento de marca: Como se mencionó en la Figura 1 el relacionamiento de marca produce algunos efectos positivos en el consumidor, entre estos están: la actitud, satisfacción, amor, apego, compra, recompra, boca a boca, lealtad, reconocimiento, elección, preferencia, entre otros. Las relaciones consumidor-marca pueden formarse con base en conexiones individuales o grupales, el primer caso, sería denominado autoconcepto, entendido como el deseo de expresar una identidad única y diferente, el segundo, puede ser la identificación de un grupo con una marca también llamada identidad grupal (Swaminathan et al., 2007; Kumar, y Kaushik, 2020). Este tipo de relaciones, denota una condición de interdependencia en la que mínimamente dos partes están vinculadas o asociadas como resultado de una serie de interacciones que influyen en el reconocimiento del consumidor por la marca de su elección. Este mecanismo permite formar vínculos e intercambio inmediato de ideas, emociones e información, puesto que se generan beneficios que sugieren un atractivo significativo para las partes (Hayes et al. 2006; Karpińska, 2016).

En relación a esto, el valor emocional que una marca tiene para un cliente es determinante en la elección del producto o servicio, ya que este último es simplemente el bien que recibe el comprador, pero la conexión sentimental que este genere es proporcionada por la marca. Esto permite entender la trascendencia de trabajar integralmente el concepto de marca y la dificultad de separarlo del producto, pues no necesariamente su percepción y valoración son iguales. La marca a diferencia del producto tiene un carácter permanente en la mente de los consumidores, aunque a través de los años ha debido adaptarse como un proceso de evolución, mantiene un contacto "imperceptible" con el sujeto que les permite a las compañías contar con una ventaja diferenciadora en el mercado. Es en este punto donde algunos autores manifiestan que el producto se compra, se usa y se evalúa; mientras que la marca se adquiere, se aprecia y se conecta con el cliente y es, a partir de este compromiso donde nace el valor de marca o brand equity (Aaker et al., 2004). 
Cuando se habla de reconocimiento o valor de marca es importante apelar a todos los sentidos, los cuales ayudan a que esta se pueda diferenciar de la competencia, en palabras de Lindstrom (2012):

Los estrategas de marketing se han concentrado desde siempre en motivar y conmover a los consumidores visualmente. La verdad es que las imágenes visuales son mucho más eficaces y más memorables cuando van de la mano con estímulos para otros sentidos como el oído o el olfato. Las compañías han comenzado a descubrir que, para lograr nuestro total compromiso emocional, no deben abrumarnos con logotipos sino bombear fragancias en nuestras narices y música en nuestros oídos (p.90).

Esto infiere que un efecto de la relación de marca viene dado por como las personas fácilmente pueden reconocer su marca predilecta a través de sus sentidos.

En cuanto al manejo de información, hay autores que manifiestan que la marca debe contar una historia para crear una conexión con su público. Para lograrlo es importante partir de hechos cotidianos que llamen la atención del consumidor, la simplicidad en este punto resulta un factor relevante que ayuda al posicionamiento de marca, lo que resulta un efecto interesante con características o elementos particulares propios de la relación, además resulta importante desligar todo el proceso comunicacional a patrones de publicidad tradicional, convirtiendo las oportunidades de interacción en actividades sencillas de forma permanente, que impulsen al público a adherirse a la marca, teniendo y haciendo un buen uso de los datos que esta relación permita (Cayolla y Loureiro, 2014; Breivik y Thorbjørnsen, 2008).

También las relaciones de marca generalmente producen dos mecanismos interesantes denominados autoexpansión e inclusión, el primero resulta cuando el consumidor requiere adquirir recursos, perspectivas e identidades que mejoren su capacidad de lograr objetivos; y el segundo que es cuando las marcas se convierten en símbolos de identidad y pueden ser altamente congruentes con la propia imagen. Estos roles difieren si la relación es nueva o antigua, lo que hace pensar que las relaciones de marca son un concepto tan complejo que se presta para múltiples análisis y perspectivas (Keller, 2012; Reimann et al., 2012). 
Otro efecto interesante es la fidelidad de marca, un concepto muy complicado de entender sino se asume el concepto de antropomorfización de la marca, puesto que funciona de igual manera como una relación de pareja, la cual desde una perspectiva humana resulta compleja de lograr sin embargo, la necesidad de tener clientes fieles ha hecho que los negocios prefieran una expansión concentrada a nivel local, permitiendo conectar directamente con los clientes y a su vez hacer seguimiento de las cosas casi a nivel individual, en perspectiva gerencial y bajo la mirada mercadológica clientes mejor cuidados significa fidelidad y mayor volumen de compras asegurados en una gran proporción (Swaminathan et al., 2007).

Por último, es importante que los estrategas de marketing, aseguren que los clientes sean capaces de identificar sus marcas, para que esto se presente se debe establecer firmemente la totalidad del significado de la marca en la mente de los consumidores al vincular estratégicamente una serie de asociaciones tangibles e intangibles con ciertas propiedades que obtengan las respuestas adecuadas de los clientes a esta identificación y que en términos de juicios y sentimientos el relacionamiento de marca se convierta en la respuesta obligada para el consumidor, creando una relación de lealtad intensa y activa entre las partes (Keller, 2012; Park et al., 2013).

\section{CONCLUSIONES}

La última década del siglo XX y los últimos años de esta revisión marcan el comienzo y la reanudación del interés por los estudios científicos sobre la relación entre una marca y los consumidores, haciendo posible elevar dicha relación como una palanca para fortalecer este campo de investigación. Esta conceptualización comienza con la idea de que los humanos antropomorfizan objetos inanimados y pueden pensar en las marcas como si fueran personas (Aaker et al., 2004) sin embargo es importante que se analice los patrones de amistad que se forman con las marcas bajo este concepto, junto con las evidencias de estas “aventuras de corta duración”, que en su momento podrían generar posibles complicaciones o malestares en los individuos.

El término relación denota una condición de interdependencia en la que las partes están vinculadas o asociadas por algún motivo, siendo esto el resultado de una serie de interacciones entre individuos y las marcas. Por ello, las empresas deben reconocer tanto diferencias como semejanzas entre los 
consumidores y proponer relaciones teniendo en cuenta las particularidades del mercado y gestionando los dos lados de la interacción presentada, aunque esta sea de un tipo especial y diferente. Visiblemente las marcas ofrecen variadas opciones para ser trabajadas, entre las más investigadas están la conciencia de marca, experiencia de marca, satisfacción de marca, imagen de marca, confianza de marca, personalidad de marca, diseño de marca, compromiso de la marca, arquitectura de marca, gestión de marca, valor percibido y la intención de uso o compra y recompra.

La arquitectura de marca es un sistema de gestión y, por tanto, su organización debe responder a la estrategia del modelo de negocio para determinado mercado. Por esta razón, para unificar la estrategia de marca muchas compañías sin tener en cuenta su estructura organizacional, optan por evaluar qué estrategia de arquitectura de marca apoyará su crecimiento y por ende afianzar su valor a futuro, lo importante en este aspecto es tener claridad en lo que se espera trasmitir al público.

El estratega de marca en su organización, debe estar al tanto de toda la información del mercado, estudiarla, analizarla y con ella planear acciones directas dirigidas a mercados objetivos específicos, acciones que por encima del "cómo" está el "saber cómo" interactuar de manera más directa con los consumidores. En otras palabras, la tarea de los gerentes de marca está en identificar de manera clara el grupo con el cual desean relacionarse, elegir los medios por los cuales ese relacionamiento sería más coherente, tener el factor diferenciador que le aporte valor a esa relación y que apelen a la variedad de herramientas que el Marketing tiene para ello.

El gerente de marca irá siempre en búsqueda de relaciones duraderas con sus mercados, lo transaccional se convierte en un objetivo ya no prioritario, sino como una resultante del ejercicio bien formulado y como parte de la respuesta a las acciones y medidas concretas, que para el caso van en búsqueda de un posicionamiento a largo plazo, pensando en el bienestar del entorno que deberá estar por encima de la venta para lograr una sustentabilidad y sostenibilidad en el tiempo.

Cuando se mencionan conceptos como conciencia, imagen e identidad de marca, se está hablando de algo que va más allá del simple diseño y construcción de un logo o de la publicidad, sino que se trabaja bajo la transmisión de ventajas, reputación, servicio y beneficios para el consumidor. 
Los diferentes análisis hechos por autores permiten identificar el soporte y el papel mediador de la confianza de marca entre las interacciones entre el consumidor y esta, además del apego emocional que impacta en la lealtad y el boca a boca positivo, hechos que permiten una “conexión amorosa”. Sin embargo, una mala conducta de la marca decepcionará casi irremediablemente las expectativas y la relación de los consumidores sobre la marca.

Este documento de revisión de la literatura existente sobre Relacionamiento de Marca es una intención de informar y describir lo que hasta el momento se ha hecho en este campo, para que encargados de marca, estrategas de marketing y docentes que tienen a su cargo temáticas relacionadas tengan una guía de lo que se ha trabajado, quienes lo han trabajado, donde lo han trabajado y de que forma se lo está trabajando hasta el mes de marzo de 2020 en el mundo entero.

\section{REFERENCIAS}

(1) Aaker, D. A. y Joachimsthaler, E. (2000). The brand relationship spectrum: The key to the brand architecture challenge. California management review, 42(4), 8-23. doi: 10.1177/000812560004200401

(2) Aaker, J., Fournier, S. y Brasel, S. A. (2004). When good brands do bad. Journal of Consumer research, 31(1), 1-16. doi: 10.1086/383419

(3) Avis, M. y Aitken, R. (2015). Intertwined: Brand personification, brand personality and brand relationships in historical perspective. Journal of Historical Research in Marketing 7(2), 208-231. doi: 10.1108/JHRM01-2014-0004.

(4) Bartels, R. (1951). Can marketing be a science? Journal of Marketing, 15(3), 319-328. doi: $10.1177 / 002224295101500306$

(5) Bengtsson, A. (2003). Towards a critique of brand relationships. Advances in Consumer Research, 30, 154154.

(6) Blackston, M. (1992). A brand with an attitude: a suitable case for treatment. Journal of the market research society, 34(3), 231-242.

(7) Blackston, M. (1993). Beyond brand personality: building brand relationships. En D. A. Aaker \& A. L. Biel (Eds.), Brand equity and advertising: Advertising's role in building strong brands (pp.113-124). Hilsdale, New Jersey: Lawrence Erlbaum. 
(8) Breivik, E. y Thorbjørnsen, H. (2008). Consumer brand relationships: an investigation of two alternative models. Journal of the Academy of Marketing Science, 36(4), 443-472. doi: 10.1007/s11747-008-0115-z

(9) Cayolla, R. y Loureiro, S. M. C. (2014). Fans club brand relationship: football passion. International Journal of Business and Globalisation 10, 12(1), 82-97.

(10) Chang, P. L. y Chieng, M. H. (2006). Building consumer-brand relationship: A cross-cultural experiential view. Psychology y Marketing, 23(11), 927-959. doi:10.1002/mar.20140

(11) Erkmen, E. y Hancer, M. (2019). Building brand relationship for restaurants: An examination of other customers, brand image, trust, and restaurant attributes, International Journal of Contemporary Hospitality Management, 31(3), 1469-1487.

(12) Fournier, S. (1998). Consumers and their brands: Developing relationship theory in consumer research. Journal of consumer research, 24(4), 343-373. doi: 10.1086/209515

(13) Hayes, J. B., Alford, B. L., Silver, L. y York, R. P. (2006). Looks matter in developing consumer-brand relationships. Journal of Product \& Brand Management, 15(5), 306-315. doi: $10.1108 / 10610420610685875$

(14) Heath, R., Brandt, D. y Nairn, A. (2006). Brand relationships: Strengthened by emotion, weakened by attention. Journal of advertising research, 46(4), 410-419. doi: 10.2501/S002184990606048X

(15) Hongcharu, B. y Eiamkanchanalai, S. (2015). Strengthening brand presence and creating brand relationships in the society through integrated marketing communications. International Journal of Economic Research, 12(4), 1307-1318.

(16) Hudson, S., Huang, L., Roth, M. S. y Madden, T. J. (2016). The influence of social media interactions on consumer-brand relationships: A three-country study of brand perceptions and marketing behaviors. International Journal of Research in Marketing, 33(1), 27-41. doi: 10.1016/j.ijresmar.2015.06.004

(17) Karpińska-Krakowiak, M. (2016). The effects of social networking sites on consumer-brand relationships. Journal of Computer Information Systems, 56(3), 204-210. doi: 10.1080/08874417.2016.1153894

(18) Kaufmann, H. R., Filho, C. G., Silva, C. R. E. y Monteiro, P. R. R. (2019). Consumer-brand relationship development in the automotive market: an integrative model. International Journal of Automotive Technology and Management, 19(3-4), 321-340.

(19) Keller, K. L. (2012). Understanding the richness of brand relationships: Research dialogue on brands as intentional agents. Journal of Consumer Psychology, 22(2), 186-190. doi: 10.1016/j.jcps.2011.11.011

(20) Knopf, J. W. (2006). Doing a literature review. PS: Political Science and Politics, 39(1), 127-132.

(21) Krake, F. (2005). Successful brand management in SMEs: a new theory and practical hints. Journal of Product \& Brand Management, 14(4), 228-238. doi: 10.1108/10610420510609230

(22) Kumar, V. y Kaushik, A. K. (2020). Building consumer-brand relationships through brand experience and brand identification. Journal of Strategic Marketing, 28(1), 39-59. doi: 10.1080/0965254X.2018.1482945 
(23) Lee, H. J. y Kang, M. S. (2013). The effect of brand personality on brand relationship, attitude and purchase intention with a focus on brand community. Academy of Marketing Studies Journal, 17(2), 85-98.

(24) Lindstrom, M. (2012). Buyology: How everything we believe about why we buy is wrong. Random House Business.

(25) Mayring, P. (2003). Qualitative Inhaltanalyse - Grundlagen und Techniken. [Qualitative content analysis]. 8th ed. Weinheim, Germany: Beltz Verlag.

(26) Nyadzayo, M. W., Matanda, M. J. y Ewing, M. T. (2016). Franchisee-based brand equity: The role of brand relationship quality and brand citizenship behavior. Industrial Marketing Management, 52, 163-174. doi: 10.1016/j.indmarman.2015.07.008

(27) Papista, E. y Dimitriadis, S. (2012). Exploring consumer-brand relationship quality and identification: Qualitative evidence from cosmetics brands. Qualitative Market Research, 15(1), 33-56. doi: $10.1108 / 13522751211191982$

(28) Park, C. W., Eisingerich, A. B. y Park, J. W. (2013). Attachment-aversion (AA) model of customer-brand relationships. Journal of consumer psychology, 23(2), 229-248. doi: 10.1016/j.jcps.2013.01.002

(29) Petromilli, M., Morrison, D. y Million, M. (2002). Brand architecture: building brand portfolio value. Strategy \& Leadership, 30(5), 22-28. doi: 10.1108/10878570210442524

(30) Reimann, M., Castaño, R., Zaichkowsky, J. y Bechara, A. (2012). How we relate to brands: Psychological and neurophysiological insights into consumer-brand relationships. Journal of Consumer Psychology, 22(1), 128-142. doi: 10.1016/j.jcps.2011.11.003

(31) Swaminathan, V., Page, K. L. y Gürhan-Canli, Z. (2007). "My” brand or “our” brand: The effects of brand relationship dimensions and self-construal on brand evaluations. Journal of consumer research, 34(2), 248259. doi: $10.1086 / 518539$

(32) Zayer, L. y Neier, S. (2011). An exploration of men's brand relationships. Qualitative Market Research, 14(1), 83-104.

(33) Zhou, Z. (2007). How to measure brand relationship quality? Frontiers of Business Research in China, 1(2), 300-318. doi: 10.1007/s11782-007-0017-9

Cómo citar este artículo: Mejía, J. y Arcos, M. (2020). Relacionamiento de marca: análisis de perspectivas y trascendencia en la gestión. Tendencias, 21(2), 242-265. https://doi.org/10.22267/rtend.202102.149 